\title{
EVALUACIÓN DE COMPETENCIAS BÁSICAS EN TIC EN DOCENTES DE EDUCACIÓN SUPERIOR EN MÉXICO.
}

\section{ASSESSMENT OF BASIC ICT COMPETENCIES IN TEACHERS OF HIGHER EDUCATION IN MEXICO.}

\author{
Dr. José Ángel Vera Noriega ${ }^{1}$ \\ avera@ciad.mx \\ Dra. Lilia Elisa Torres Moran² \\ 1torres@itson.mx \\ MDR Edgar Emmanuel Martínez García ${ }^{1}$ \\ max_021@hotmail.com
}
${ }^{(1)}$ Centro de Investigación en Alimentación y Desarrollo, A.C. Departamento de desarrollo humano y bienestar social. Carretera a la Victoria Km .6 Ejido La Victoria, 1735, 83000, Hermosillo, Sonora (México)
${ }^{(2)}$ Instituto Tecnológico de Sonora. Coordinación de comunicación institucional. Calle 5 de febrero, 418 sur, Colonia Centro, 85000, Obregón, Sonora (México)

Este estudio evaluó las competencias básicas en TIC de docentes en Educación Superior en México, la muestra se integró por 432 docentes. Los datos se obtuvieron a través de un instrumento de medida basado en el modelo "Estándares UNESCO de Competencias en TIC para Docentes 2008". Entre los resultados destacó que los docentes poseen un dominio de moderado a moderado alto de las TIC, asimismo, después de los análisis ANOVA y las pruebas $t$ de Student, se encontró que los factores como correo electrónico, Internet, edad y adopción basada en intereses modifican el nivel de dominio de las Tecnologías de la Información y Comunicación.

Palabras clave: Educación Superior, evaluación por competencias, alfabetización digital, tecnologías de la información y la comunicación (TIC).

This study assessed the basic ICT competencies in Higher Education in Mexico; the sample was composed of 432 teachers. Data were obtained through a measuring instrument based on the model "UNESCO Competency Standards on ICT for Teachers 2008". Amongst its outcomes, it highlights that the teachers master the ICT in a moderate to a moderately high manner. In addition, after ANOVA analysis and Student t tests, it was found that factors such as E-mail, Internet, age and adoption based on interests, modify the level of mastery towards the Information and Communication Technology.

Keywords: Higher Education, teacher's competencies, information literacy, information and communication technologies (ICT).

Píxel-Bit. Revista de Medios y Educación. $N^{o}$ 44. Enero 2014. ISSN: 1133-8482.

E-ISSN: 2171-7966. doi: http://dx.doi.org/10.12795/pixelbit.2014.i44.10 


\section{Introducción.}

La educación basada en competencias es una orientación educativa que pretende dar respuestas a la sociedad del conocimiento. En la actualidad la educación necesita una visión congruente con las competencias que las instituciones de educación forzosamente requieren para que los docentes, a través de la práctica pedagógica, puedan confrontar las exigencias en el siglo XXI. En este marco, la evaluación de las competencias determina aquello específico que se va a desempeñar o construir y se basa en la comprobación de la capacidad de construirlo o desempeñarlo (Argudín, 2005).

Las competencias básicas se definen como la "capacidad de poner en marcha de manera integrada conocimientos adquiridos y rasgos de personalidad que permiten resolver situaciones diversas" (Marquès, 2000, p. 7), incluyen tanto los saberes o conocimientos teóricos como habilidades o conocimientos prácticos y también las actitudes y compromisos personales. Por lo tanto, son formas de combinar los recursos personales para realizar una tarea y van más allá del saber y saber hacer o aplicar porque incluyen también el saber ser o estar, lo que implica el desarrollo de capacidades, no sólo la adquisición de contenidos puntuales y descontextualizados, y suponen la capacidad de usar funcionalmente los conocimientos y habilidades en contextos diferentes para desarrollar acciones no programadas previamente.

Por ello, según Marquès (2000), desde la perspectiva de las capacidades, un conocimiento tiene valor educativo cuando contribuye al desarrollo personal del estudiante; desde la perspectiva de las competencias, el conocimiento debe contribuir a la realización de una tarea. En este contexto, para evaluar las competencias hay que centrarse en la realización de actividades, pues las mismas posibilitan que el dominio del contenido, se transforme en competencia.

En el nuevo paradigma educativo adquiere singular importancia el uso de las TIC por parte de los docentes, así como sus competencias para desarrollar nuevos modos de aprender. Los rasgos característicos de este modelo educativo exigen el desarrollo de un perfil profesional, de roles y competencias diferentes a las tradicionales en los estudiantes y los docentes en donde es un requisito básico la profesionalización de los últimos, lo que implica la exigencia de una formación pedagógica institucionalizada y sistemática, cuya finalidad sea la de facilitar el aprendizaje de nuevas competencias docentes (Escudero, 1997).

Los procesos de innovación respecto a la utilización de las TIC en la docencia universitaria suelen partir, la mayoría de las veces, de las disponibilidades y soluciones tecnológicas existentes en la infraestructura de la Universidad. Hay que tener presente que, como cualquier innovación educativa, estamos ante un proceso con múltiples facetas: en él intervienen factores políticos, económicos, ideológicos, culturales y psicológicos, y afecta a diferentes planos contextuales, desde el nivel del aula hasta el del grupo de Universidades (Consejo Federal de Educación, 2010).

Sin embargo, abordar las TIC desde la perspectiva de la innovación pedagógica es una oportunidad para reposicionar a los docentes a partir de su conocimiento didáctico. El dominio de las TIC por parte de los docentes es solo un paso inicial, ya que la esencia consiste en incorporar los aportes 
de las TIC para poner en marcha nuevas estrategias de aprendizaje que sumen valor a la práctica educativa cotidiana. Explorar las posibilidades de las TIC para provocar cambios en las prácticas que permitan mayor y mejor apropiación de contenidos por parte de estudiantes, a la vez que aporten al desarrollo de nuevas capacidades que les permitan a los mismos situarse a la altura de los nuevos desafíos.

El uso de las TIC en la Educación Superior permite el desarrollo de proyectos innovadores que posibiliten los cambios de actitudes, pensamientos, culturas contenidos, modelos de trabajo y sentidos de las prácticas pedagógicas. Estas innovaciones generan nuevos ambientes educativos que inciden sobre la redefinición curricular, estrategias de enseñanza y de aprendizaje, modelos didácticos, dinámicas del aula y cambios en la organización institucional.

En relación a lo anterior, Salinas (2004) sostiene que las instituciones educativas están comprometidas en revisar sus referentes actuales y promover experiencias innovadoras en procesos de enseñanzaaprendizaje apoyados en las TIC. En este terreno las Universidades convencionales se enfrentan a dificultades asociadas a la capacidad de flexibilización de su estructura organizacional.

El manejo de las TIC ayuda a docentes y estudiantes a adquirir las capacidades necesarias para llegar a ser: a) competentes para utilizar tecnologías de la información; b) buscadores, analizadores y evaluadores de la información; c) solucionadores de problemas y tomadores de decisiones; d) usuarios creativos y eficaces de herramientas de productividad; e) comunicadores, colaboradores, publicadores y productores; y f) ciudadanos informados, responsables y capaces de contribuir al desarrollo social (UNESCO, 2008).

Por su parte, López (2003) señala que un buen desarrollo profesional por sí solo no es efectivo, la cultura organizacional de los sistemas educativos debe cambiar, esto implica desarrollar cultura de trabajo colaborativo o comunidades de aprendizaje profesional. Para este autor las nuevas exigencias asociadas al aprovechamiento de las TIC en la producción y los servicios, y la necesidad de potenciar el desarrollo de habilidades de acceso y uso de la información en ambientes digitales de trabajo, modifica sensiblemente los procesos que giran en torno a ello, repercutiendo también en el diseño de los procesos de formación en las instituciones educativas.

Para Rodés (2011), resulta fundamental ofrecer modelos organizativos de integración de TIC como insumo para la toma de decisiones y la planificación en las Universidades, estableciendo niveles de liderazgo institucional desde la perspectiva de los actores, de modo que la innovación tenga carácter apropiado a la idiosincrasia y estilo institucional. El conocimiento generado ofrece modelos de desarrollo en la temática con carácter local, permitiendo su transferencia a otros casos similares en la región.

De acuerdo con Lasnier (2000), competencia es un saber hacer complejo, producto de la integración, movilización y adecuación de las capacidades y habilidades y de conocimientos que usados en situaciones parecidas no generalizables permiten la consecución de los objetivos. Así, una competencia como saber hacer de cierta complejidad, está integrada por capacidades (las cuales exigen conocimientos procedimentales y condicionales). A su vez 
las capacidades están constituidas por habilidades necesarias para una ejecución compleja.

Lasnier (2000), considera que las exigencias de innovación docente y la aparición de las TIC, comprenden la adaptación de los programas antiguos por objetivos a programas por competencias y subordinación de los contenidos a dichas competencias que, conceptualmente, son un saber hacer complejo e integrador; lo que implica un cambio sustancial en los métodos de enseñanza y aprendizaje, que en esta nueva situación pasan de ser generalmente centrados en el docente, a centrarse en los estudiantes, en donde el docente habrá de buscar situaciones de aprendizaje contextualizadas, y focalizadas al desarrollo en los estudiantes y de su capacidad de aplicación y resolución de problemas.

Ortega (2008) nos indica que la formación docente facilitará el uso de las TIC y su integración al currículo en los centros educativos, considerando que en los nuevos entornos virtuales de enseñanza-aprendizaje, el docente juega un papel de tutor y mediador cuyo desempeño impacta en el logro de los objetivos de aprendizaje. Para Marín, Vázquez, Llorente y Cabero (2012), la necesidad de capacitación se refleja en los conocimientos y utilidades de las herramientas TIC que el docente tiene a su alcance, en consecuencia se puede generalizar la necesidad de una formación específica en determinadas herramientas como los sistemas de e-learning y de videoconferencia. Asimismo, los docentes reflejan que tienen un conocimiento moderado en estas áreas telemáticas, no son expertos en su aplicación para su propio desarrollo personal, tanto a nivel formativo, como a nivel de participación en comunidades virtuales con objetivos sociales y de aprendizaje.

Bosco (2008) coincide con Tejada (2009), en que los retos de la sociedad de conocimiento, hacen necesario incorporar a la formación docente universitaria las nuevas competencias para el ejercicio de la docencia. Dichas competencias implican el uso de las TIC en la enseñanza y el aprendizaje en base a una comprensión crítica que permita al docente integrarlas a una secuencia didáctica orientada al logro de objetivos pedagógicos.

En este sentido, estudios realizados por Pérez, Álvarez, del Moral y Pascual, (1998), Fernández y Cebreiro (2002), encontraron que el docente en el siglo XXI ha visto cambiado el rol que habitualmente había desempeñado. Las TIC crean nuevos entornos que establecen la necesidad de innovación en relación a las formas que interactúan docentes y alumnos en el aula.

Esta situación pone de manifiesto la existencia de necesidades de formación profesional y actualización docente en el uso de las TIC integradas al currículo. Por ello se hace necesario señalar como un problema la necesidad de diseñar medidas confiables y validas pero además proponer metodologías analíticas que nos proporcionen datos para la mejora continua en la formación del profesorado en el manejo de TIC en las universidades.

\section{Metodología.}

El objetivo del presente estudio es conocer y analizar la competencia básica de los profesores de Educación Superior en el uso de las TIC en los procesos de enseñanza. 


\begin{tabular}{|l|c|c|c|}
\hline DIRECCIONES ACADÉMICAS & $\begin{array}{c}\text { NÚMERO DE } \\
\text { DOCENTES }\end{array}$ & $\begin{array}{c}\text { PORCENTAJE } \\
\text { DE DOCENTES }\end{array}$ & $\begin{array}{c}\text { DOCENTES } \\
\text { PARA LA } \\
\text { MUESTRA }\end{array}$ \\
\hline Dirección de Recursos Naturales & 152 & 11.58 & 50 \\
\hline Dirección de Ingeniería y Tecnología & 311 & 29.87 & 129 \\
\hline $\begin{array}{l}\text { Dirección de Ciencias Sociales y } \\
\text { Humanidades }\end{array}$ & 151 & 34.02 & 147 \\
\hline $\begin{array}{l}\text { Dirección de Ciencias Económicas y } \\
\text { Administrativas }\end{array}$ & 308 & & 106 \\
\hline
\end{tabular}

Tabla 1. Población de docentes de la Universidad Pública Mexicana.

\subsection{Condiciones de muestreo.}

Para estos fines, se contó con una población total de 922 docentes de la UPM (Tabla 1). Se conformó una muestra representativa de 432 sujetos voluntarios de las distintas áreas del conocimiento de la UPM, a partir de la fórmula de Sierra (1998). Ésta muestra fue diseñada con un $95 \%$ de confiabilidad, $3 \%$ de error y una $\mathrm{p}=.5 \mathrm{y} \mathrm{q}=.5$.

Los participantes fueron docentes de ambos sexos y diferentes áreas de formación. Los mismos están adscritos con diferentes estatus de contratación: definitivos, interinos y de tiempo parcial, que se distribuyeron de manera ponderada por división académica con diferentes perfiles e interés con relación al uso de las TIC.

\subsection{Descripción del instrumento.}

En la recolección de datos se utilizó un cuestionario para evaluar las competencias básicas en TIC desarrollado bajo los lineamientos de UNESCO (2008). El cuestionario se responde en una sola aplicación y está dividido en cuatro partes, cada una con un fin específico a saber:

La primera dimensión, Variables personales, comprende 14 reactivos que se refieren a la recopilación de la información general de los sujetos participantes en el estudio (reactivos 1-14).

- La segunda dimensión, Adopción basada en intereses, incluye una pregunta que evalúa la adopción de las tecnologías basada en intereses que ubica al docente en un continuo de ocho niveles de especialización en el manejo de las TIC, donde la respuesta se elige del menor al mayor nivel de especialización, según el caso (reactivo 15).

- Adicionalmente la tercera dimensión, Evaluación de manejo de TIC, comprende 35 reactivos y profundiza en el conocimiento que expresa tener el colectivo docente sobre la existencia y estado de uso de los diversos equipos y materiales tecnológico-didácticos existentes en su centro de trabajo y conocer las actitudes de los mismos relacionadas con la colaboración en la mejora de la organización de los medios y los recursos tecnológicos. De la misma forma, estas preguntas constituyen una medida de evaluación de 


\begin{tabular}{c|c|c|c|c|c|c|c|c|c|c|c|c|c|}
\hline Edad & $\mathbf{N}$ & Esc & $\mathbf{n}$ & $\mathbf{G}$ & $\mathbf{n}$ & $\begin{array}{c}\text { Uso de Pc } \\
\text { en clase }\end{array}$ & $\mathbf{N}$ & $\begin{array}{c}\text { Tiempo } \\
\text { enseñando }\end{array}$ & $\mathbf{N}$ & $\begin{array}{c}\text { Universo } \\
\text { Científico }\end{array}$ & $\mathbf{N}$ & $\begin{array}{c}\text { Adopción } \\
\text { basada }\end{array}$ & $\mathbf{n}$ \\
\hline $21-29$ & 124 & L & 144 & M & 222 & 0 a 3veces & 98 & menos 1 año & 39 & $\begin{array}{c}\text { Ciencias } \\
\text { duras }\end{array}$ & 151 & N-B & 120 \\
\hline $30-39$ & 145 & M & 258 & F & 210 & 4 a 5 veces & 93 & 1 a 3 años & 112 & $\begin{array}{c}\text { Ciencias } \\
\text { blandas }\end{array}$ & 281 & N-R & 168 \\
\hline $40-49$ & 102 & D & 30 & & & $\begin{array}{c}\text { Más de 5 } \\
\text { veces }\end{array}$ & 241 & 3 a 5 años & 59 & & & N-A & 144 \\
\hline $50-+55$ & 61 & & & & & & & 5 o más & 222 & & & & \\
\hline
\end{tabular}

Tabla 2. Características de la muestra.

Nota. $\mathrm{n}=$ Muestra, Esc $=$ Escolaridad, $\mathrm{G}=$ Género, $\mathrm{L}=$ Licenciatura, $\mathrm{M}=$ Maestría, $\mathrm{D}=$ Doctorado, $\mathrm{N}-\mathrm{B}=$ Nivel Básico, N-R= Nivel Refinamiento, N-A=Nivel Avanzado

competencias básicas, cuyas dimensiones son: instrumentos básicos y tratamiento de la información y comunicación (reactivos 1650).

- Finalmente, la cuarta dimensión, Aplicación educativa, comprende 14 reactivos que evalúan planeación educativa y procesos de ejecución para obtener el nivel de cumplimiento de las competencias de la integración de las TIC al proceso de enseñanza-aprendizaje (reactivos 51-64).

Las características psicométricas de la medida de evaluación de dominio (parte 3) y aplicación educativa (parte 4) quedan de la siguiente manera: a) evaluación de dominio de TIC (parte 3), está compuesta por dos dimensiones que son hardware y manejo de sistema operativo con 21 reactivos, de la cual todos los reactivos pertenecen al manejo elemental del equipo y del sistema operativo con una $\alpha$ de Cronbach de .96; b) una segunda dimensión es el uso preliminar de software con 14 reactivos y un alfa de
Cronbach de .74 y c) aplicación educativa (parte 4) se presenta como una sola dimensión con 14 reactivos y una? de Cronbach de .92 . A partir de la sumatoria de estas tres dimensiones se construyó una variable llamada total de dominio (Vera \& Torres, 2012).

\section{Análisis de datos y resultados.}

La muestra estuvo conformada por 432 docentes participantes con las siguientes características: dominan docentes menores de 40 años, el grado de escolaridad es primordialmente de Maestría, le siguen Licenciatura y finalmente Doctorado. El 51\% de los sujetos participantes corresponde al género masculino; el mayor número cuenta con cinco o más años impartiendo clases en el nivel universitario. Además, el 39\% de los encuestados respondieron que usan las TIC en sus clases. En la Tabla 2 se puede apreciar detalladamente las características de la muestra.

Píxel-Bit. Revista de Medios y Educación. No 44. Enero 2014. ISSN: 1133-8482.

E-ISSN: 2171-7966. doi: http://dx.doi.org/10.12795/pixelbit.2014.i44.010 
En relación con el nivel de cumplimiento de los criterios de evaluación para el manejo de la tecnología de la información y la comunicación para su aplicación a los objetivos de su plan en clase, se compararon los factores dicotómicos sobre el uso de email (correo electrónico), Internet, capacitación y universo científico de pertenencia del docente, a través de una prueba $t$ de Student para datos independientes con las variables de dominio: manejo de hardware, aplicación educativa y uso de software.

Los resultados indican que el $87 \%$ de los docentes usan el correo electrónico de cuatro a cinco veces a la semana; el $89 \%$ de los docentes utiliza el Internet más de cinco veces a la semana (Tabla 3). En la respuesta de los ítems referidos al factor capacitación, resultó que el $74 \%$ de los docentes ha recibido capacitación en el manejo de las TIC y el factor de universo científico indica que el $65 \%$ pertenecen al área de ciencias blandas. Los participantes con las tasas más altas de manejo hardware, uso software y total dominio coincide con aquellos que utilizan el correo electrónico de 4 a 5 veces a la semana, comparado con aquellos que lo usan de 0 a 3 veces a la semana. Igualmente, el contraste de $t$ de Student mostró que el uso de e-mail e Internet impactan el total dominio en el manejo de hardware y uso de software y no establecen diferencias estadísticamente significativas en la aplicación educativa. Sin embargo, la comparación entre el grupo que ha recibido capacitación y el que reporta no haberla recibido presenta diferencias estadísticamente significativas para la variable aplicación educativa y uso de software (Tabla 3).

$\mathrm{n}=$ muestra, desv. típ. = desviación típica.
Se compararon factores politómicos sobre adopción de la tecnología, edad en rangos, uso de la computadora, uso de procesadores de palabras y uso de hoja de cálculo, a través de una prueba ANOVA simple sólo con las variables manejo de hardware, aplicación educativa, uso de software y total dominio.

Para la interpretación de las medias en las dimensiones de dominio los valores van de uno a cinco. Los valores superiores de cuatro a cinco se interpretan como dominios altos, de tres a cuatro como dominio moderado, de dos a tres dominios bajo y de uno a dos dominios insuficientes. En las dimensiones de actitud digital se asumen valores promedio de uno a siete, donde de cuatro a cinco se interpreta como actitud positiva baja; de cinco a seis se interpreta como positiva moderada, de seis a siete se interpreta actitud positiva alta y para valores menores se considera de uno a dos actitud negativa alta, de dos a tres actitud negativa moderada y de tres a cuatro es considerada actitud negativa baja.

En el factor de la frecuencia con la que utiliza computadora, resulta que un $55 \%$ de los docentes tiene una frecuencia mayor de uso, equivalente a 5 veces o más a la semana. Todos los análisis de varianza (ANOVA) simple para la comparación de los grupos asociados a los niveles de los factores resultaron con diferencias estadísticamente significativas al .01 incluyendo los siguientes: adopción en rangos, edad en rangos, utilización de computadora, procesador de palabras y hoja de cálculo (Tabla 4).

Tabla 4. Análisis de varianza simple para las dimensiones de la variable total de dominio y los factores frecuencia de uso de computadora, procesador de palabras, hoja de cálculo, edad y adopción de una muestra de docentes. (432) de una UPM. Nota. $n=$ muestra, $\mathrm{F}=$ Estadístico de contraste. 


\begin{tabular}{|c|c|c|c|c|c|c|c|c|c|c|c|c|c|}
\hline & & \multirow{2}{*}{\multicolumn{3}{|c|}{$\begin{array}{c}\text { MANEJO DE } \\
\text { HARDWARE } \\
\text { Media/desv. típ./ } t\end{array}$}} & \multicolumn{3}{|c|}{$\begin{array}{l}\text { APLICACIÓN } \\
\text { EDUCATIVA }\end{array}$} & \multicolumn{3}{|c|}{$\begin{array}{c}\text { USO DE } \\
\text { SOFTWARE }\end{array}$} & \multicolumn{3}{|c|}{$\begin{array}{c}\text { TOTAL } \\
\text { DOMINIO }\end{array}$} \\
\hline & & & & & \multicolumn{3}{|c|}{ Media/desv. típ./ $t$} & \multicolumn{3}{|c|}{ Media/desv. típ./t } & \multicolumn{3}{|c|}{ Media/desv. típ. / $t$} \\
\hline \multicolumn{14}{|l|}{ e-mail } \\
\hline $0-3$ veces & 53 & 4.20 & $(.83)$ & & 3.64 & (1.07) & & 3.47 & $(1.14)$ & & 3.76 & $(.95)$ & \\
\hline $4-5$ veces & 379 & 4.61 & $(.51)$ & -4.97 & 3.92 & $(.82)$ & -2.20 & 3.88 & $(.86)$ & 3.16 & 4.15 & $(.65)$ & -3.75 \\
\hline \multicolumn{14}{|l|}{ Internet } \\
\hline $0-5$ veces & 7 & .18 & $(.84)$ & & .75 & $(1.04)$ & & .49 & $(1.16)$ & & .79 & $(.96)$ & \\
\hline Más de 5 veces & 85 & .61 & $(.52)$ & 4.87 & .90 & $.83)$ & 1.13 & .87 & $.86)$ & 2.72 & .14 & (.66) & 3.26 \\
\hline \multicolumn{14}{|l|}{ Capacitación } \\
\hline No & 15 & .48 & $(.64)$ & & .63 & $.91)$ & & .57 & $.98)$ & & .91 & $(.77)$ & \\
\hline $\mathrm{Si}$ & 17 & .59 & $(.55)$ & 1.60 & .97 & $.82)$ & 3.70 & .93 & $.83)$ & 3.70 & .17 & (.67) & 3.37 \\
\hline \multicolumn{14}{|l|}{ Universo científico } \\
\hline Ciencias duras & 51 & .66 & $(.47)$ & & .05 & $(.70)$ & & .13 & $(.71)$ & & .30 & $(.56)$ & \\
\hline Ciencias blandas & 81 & .51 & $(.62)$ & .68 & .79 & $(.92)$ & .90 & .67 & $(.96)$ & .13 & .00 & $(.75)$ & .27 \\
\hline
\end{tabular}

Tabla 3. Análisis de t de Student para muestras independientes de las dimensiones de la variable total de dominio y los factores frecuencia de uso de e-mail, Internet, capacitación y universo científico de una muestra de docentes (432) de la UPM.

Como se aprecia en la Tabla 4, con relación al factor adopción de la tecnología basada en intereses (adopción en rangos) el 39\% de los docentes se auto-perciben en el nivel refinamiento, el 33\% dentro del avanzado y el $28 \%$ en el básico de acuerdo con los resultados, el procesador de palabras está siendo utilizado por el $60 \%$ de los docentes con una frecuencia de más de cinco veces a la semana.

\section{Conclusiones.}

Los datos sobre evaluación del manejo de las TIC para la aplicación a los objetivos del plan de clase, indican que la mayoría de los docentes usan el correo electrónico e Internet en un promedio equivalente a cuatro veces por semana. En relación al manejo de hardware y uso de software se obtuvieron valores altos en comparación con aplicación educativa; es decir, los docentes manejan más el hardware y usan el software para otras actividades fuera del aula.

Para las variables dicotómicas, capacitación y tipo de universo científico, las variables aplicación educativa y uso de software resultaron con mayor diferenciación, lo que significa que el uso didáctico de las tecnologías, está relacionado con las 
necesidades que imponen los diferentes universos científicos (Ciencias duras y Ciencias blandas). Por otro lado, los docentes que están capacitados en TIC presentan diferencias significativas en la planeación educativa, comparados con aquellos sin capacitación, lo cual se traduce en un mayor dominio de las tecnologías de la información en relación a la elaboración de materiales de apoyo en las clases.

El hallazgo anterior reafirma lo señalado por Bosco (2008) y Rosario y Vásquez (2012), quien plantea que los retos de la sociedad del conocimiento hacen necesario incorporar el uso de las TIC en la formación docente para el ejercicio de nuevas competencias en la tarea pedagógica. Según este autor, dichas competencias implican el diseño y desarrollo de cursos de capacitación sobre el uso de las TIC en la enseñanza y el aprendizaje, en base a una comprensión crítica que permita al docente integrarlas a una secuencia didáctica orientada al logro de objetivos pedagógicos.

Los resultados evidencian que existe relación entre el nivel de adopción y el dominio de las habilidades asociadas a la aplicación educativa, lo que significa que los sujetos que se auto-perciben dentro del nivel avanzado tienen mayor dominio en la aplicación de las tecnologías dentro del proceso enseñanza-aprendizaje.

Las magnitudes de diferencias más bajas se encuentran dentro de la variable de uso de software y la más alta para aplicación educativa lo cual muestra que en la medida que el docente se percibe más competente en TIC su puntaje en aplicación educativa aumenta. El resultado del factor de uso de la computadora por parte del docente indica que a mayor frecuencia del uso de la computadora por semana, aumenta su percepción de autodominio en la aplicación de las TIC en el aula.

Lo anterior coincide con la afirmación de Cabero (2011), quien en su estudio encontró que el docente se auto-percibe capacitado para el uso de las TIC en las aulas, dado que manifiesta conocer diversas aplicaciones ofimáticas para su trabajo dentro de clase. Sin embargo, en la realidad presenta una baja formación o capacitación digital en herramientas concretas, como por ejemplo diseñar actividades online que complementen o apoyen los procesos de enseñanza y aprendizaje, por lo que se infiere la necesidad de una formación específica en estas áreas telemáticas.

Con respecto al factor edad, se presentó el mismo comportamiento en los análisis subsecuentes, en el sentido de que a menor edad del docente se percibe con mayor dominio de las TIC y a mayor edad disminuye en sus conocimientos en relación a su uso. Este hallazgo muestra la necesidad de poner especial atención en la capacitación del grupo de docentes de 50 años o más, elaborar programas de capacitación diseñados exclusivamente para este perfil docente y lograr así que se sientan motivados en sus tareas de enseñanza-aprendizaje considerando que son poseedores de una experiencia valiosa para la formación de los estudiantes dentro de la institución.

Las afirmaciones coinciden con los resultados de Sanabria (2006) y Rangel y Peñalosa (2013), quien sostiene la importancia de la integración curricular de las TIC como un proceso de cambio e innovación educativa. De la misma manera considera que la formación del profesorado es una variable relevante que influye, tanto en las formas de uso e integración de estas tecnologías en los 
contextos del aula como en los procesos de cambio e innovación educativa.

Atendiendo al grado de cumplimiento de los criterios de evaluación del manejo de las TIC, su relación con frecuencia de uso y actitudes, los resultados indican que los docentes que hacen uso de correo electrónico de cuatro a cinco veces a la semana, presentan mayor frecuencia en la variable dominio, con una autopercepción de mayor desempeño en el uso de las TIC en el proceso de enseñanza-aprendizaje.

El análisis ANOVA presentó coincidencia en la relación entre los niveles de los factores de adopción de las TIC, basada en intereses, utilización de computadora, utilización del procesador de palabras y utilización de la hoja de cálculo, los cuales al presentar incrementos en frecuencia provocan aumento en los valores de media correspondientes a las variables de dominio de las TIC y actitud digital. El factor edad se comporta de manera distinta, ya que a medida que éste aumenta hay un descenso en el dominio y actitud hacia las TIC.

En este sentido, Ortega (2008) sostiene que la formación docente facilitará el uso de las TIC y su integración al currículo en los centros educativos, considerando que en los nuevos entornos virtuales de enseñanzaaprendizaje el docente juega un papel de tutor y mediador y cuyo desempeño impacta el logro de los objetivos de aprendizaje.

Tejada (2009) considera que las competencias tecnológicas que debe poseer el futuro docente han de orientarse a potenciar la integración curricular de las TIC, concebida esta como su uso cotidiano, ético, legal y responsable. Por lo tanto, la competencia digital clave del docente, entendida como capacidad de realizar actos profesionales con resultados reconocibles en el mundo de la educación según dicho autor es $<$ saber utilizar e incorporar adecuadamente en las actividades de enseñanza-aprendizaje a las tecnologías de la información y la comunicación »> (p. 4). Esto quiere decir que la formación del profesorado no puede reducirse sólo a la adquisición de competencias digitales o destrezas tecnológicas, sino que debe basarse en su aplicación didáctica. Con carácter general el docente ha de planificar, impartir, asesorar y evaluar acciones formativas, elaborando $\mathrm{y}$ utilizando medios y recursos didácticos, promoviendo la calidad de la formación y la actualización didáctica.

Lo anterior coincide con Escudero (1997) quien sostiene que la perspectiva tecnológica sobre la innovación educativa, enaltece las potencialidades educativas e innovadoras de las tecnologías, convirtiéndose en motor del cambio educativo, y en consecuencia, es necesario atender a la dotación de recursos tecnológicos a los centros educativos, la formación de alumnos y profesores en el dominio de las habilidades técnicas para el manejo de las mismas. Desde la perspectiva innovación educativa, este autor resalta que las TIC son un componente más de un programa educativo que es necesario contextualizar en las coordenadas teóricas y prácticas de la realidad cultural de las escuelas y de los docentes, y por tanto, definen la integración de las TIC como un proceso complejo de cambio e innovación educativa que no se puede reducir a la dotación de equipos a los centros educativos y a la formación de profesores y alumnos como simples usuarios de estas tecnologías. 


\begin{tabular}{|c|c|c|c|c|c|c|c|c|c|c|}
\hline \multirow[b]{2}{*}{ CATEGORÍAS } & & \multirow[b]{2}{*}{$n$} & \multicolumn{2}{|c|}{$\begin{array}{l}\text { MANEJO DE } \\
\text { HARDWARE }\end{array}$} & \multicolumn{2}{|c|}{$\begin{array}{l}\text { APLICACIÓN } \\
\text { EDUCATIVA }\end{array}$} & \multicolumn{2}{|c|}{$\begin{array}{c}\text { USO DE } \\
\text { SOFTWARE }\end{array}$} & \multicolumn{2}{|c|}{$\begin{array}{c}\text { TOTAL } \\
\text { DOMINIO }\end{array}$} \\
\hline & & & Media & $\mathbf{F}$ & Media & $\mathbf{F}$ & Media & $\mathbf{F}$ & Media & $\mathbf{F}$ \\
\hline \multirow[t]{3}{*}{$\begin{array}{l}\text { Adopción en } \\
\text { rangos }\end{array}$} & 1: Básico & 20 & 4.22 & \multirow[t]{3}{*}{32.77} & 3.27 & \multirow[t]{3}{*}{64.12} & 3.25 & \multirow[t]{3}{*}{45.05} & 3.61 & \multirow[t]{3}{*}{56.30} \\
\hline & 2: Refinamiento & 68 & 4.65 & & 3.94 & & 3.92 & & 4.18 & \\
\hline & 3: Avanzado & 44 & 4.73 & & 4.32 & & 4.21 & & 4.42 & \\
\hline \multirow[t]{4}{*}{ Edad en rangos } & $1: 21-29$ & 24 & 4.69 & \multirow{4}{*}{13.14} & 4.05 & \multirow{4}{*}{7.95} & 3.98 & \multirow{4}{*}{10.70} & 4.26 & \multirow{4}{*}{13.23} \\
\hline & $2: 30-39$ & 45 & 4.63 & & 3.94 & & 3.97 & & 4. 2 & \\
\hline & $3: 40-49$ & 02 & 4.54 & & 3.87 & & 3.78 & & 4.06 & \\
\hline & $4: 50-55+$ & 1 & 4.17 & & 3.42 & & 3.28 & & 3.62 & \\
\hline \multirow[t]{3}{*}{$\begin{array}{l}\text { Utiliza } \\
\text { computadora }\end{array}$} & 1:0-3 veces & 8 & 4.18 & \multirow{3}{*}{30.59} & 3.33 & \multirow{3}{*}{31.13} & 3.27 & \multirow{3}{*}{27.98} & 3.62 & \multirow{3}{*}{34.87} \\
\hline & 2: 4-5 veces & 3 & 4.66 & & 3.91 & & 3.88 & & 4.18 & \\
\hline & $\begin{array}{l}\text { 3: } 5 \text { veces o } \\
\text { más }\end{array}$ & 41 & 4.09 & & 4.09 & & 4.04 & & 4.27 & \\
\hline \multirow{3}{*}{$\begin{array}{l}\text { Utiliza } \\
\text { procesador de } \\
\text { palabras }\end{array}$} & 1:0-3 veces & 7 & 4.24 & \multirow{3}{*}{15.79} & 3.49 & \multirow{3}{*}{12.16} & 3.37 & \multirow{3}{*}{14.98} & 3.71 & \multirow{3}{*}{17.13} \\
\hline & $2: 4-5$ veces & 4 & 4.56 & & 3.83 & & 3.77 & & 4.07 & \\
\hline & $\begin{array}{l}3: 5 \text { veces o } \\
\text { más }\end{array}$ & 61 & 4.65 & & 4.02 & & 3.99 & & 4.23 & \\
\hline \multirow[t]{4}{*}{$\begin{array}{l}\text { Utiliza hoja de } \\
\text { cálculo }\end{array}$} & 1: $0-1$ vez & 07 & 4.26 & \multirow{4}{*}{14.85} & 3.51 & \multirow{4}{*}{11.62} & 3.22 & \multirow{4}{*}{27.94} & 3.66 & \multirow{4}{*}{23.16} \\
\hline & 2: $2-3$ veces & 18 & 4.59 & & 3.88 & & 3.88 & & 4.13 & \\
\hline & 3: $4-5$ veces & 2 & 4.68 & & 3.99 & & 4.02 & & 4.25 & \\
\hline & $\begin{array}{l}\text { 4: } 5 \text { veces o } \\
\text { más }\end{array}$ & 25 & 4.71 & & 4.14 & & 4.19 & & 4.36 & \\
\hline
\end{tabular}

Tabla 4. Análisis de varianza simple para las dimensiones de la variable total de dominio y los factores frecuencia de uso de computadora, procesador de palabras, hoja de cálculo, edad y adopción de una muestra de docentes. (432) de una UPM. Nota. $n=$ muestra, $F=$ Estadístico de contraste. 


\begin{tabular}{|c|c|c|c|c|c|c|c|c|c|c|}
\hline \multirow[b]{2}{*}{ CATEGORÍAS } & & \multirow[b]{2}{*}{$n$} & \multicolumn{2}{|c|}{$\begin{array}{l}\text { MANEJO DE } \\
\text { HARDWARE }\end{array}$} & \multicolumn{2}{|c|}{$\begin{array}{l}\text { APLICACIÓN } \\
\text { EDUCATIVA }\end{array}$} & \multicolumn{2}{|c|}{$\begin{array}{c}\text { USO DE } \\
\text { SOFTWARE }\end{array}$} & \multicolumn{2}{|c|}{$\begin{array}{l}\text { TOTAL } \\
\text { DOMINIO }\end{array}$} \\
\hline & & & Media & $\mathbf{F}$ & Media & $\mathbf{F}$ & Media & $\mathbf{F}$ & Media & $\mathbf{F}$ \\
\hline \multirow[t]{4}{*}{$\begin{array}{l}\text { Utiliza hoja de } \\
\text { cálculo }\end{array}$} & 1:0-1 vez & 07 & 4.26 & \multirow{4}{*}{14.85} & 3.51 & \multirow{4}{*}{11.62} & 3.22 & \multirow{4}{*}{27.94} & 3.66 & \multirow{4}{*}{23.16} \\
\hline & 2: 2-3 veces & 18 & 4.59 & & 3.88 & & 3.88 & & 4.13 & \\
\hline & 3: $4-5$ veces & 2 & 4.68 & & 3.99 & & 4.02 & & 4.25 & \\
\hline & $\begin{array}{l}\text { 4: } 5 \text { veces o } \\
\text { más }\end{array}$ & 25 & 4.71 & & 4.14 & & 4.19 & & 4.36 & \\
\hline
\end{tabular}

Tabla 4. Análisis de varianza simple para las dimensiones de la variable total de dominio y los factores frecuencia de uso de computadora, procesador de palabras, hoja de cálculo, edad y adopción de una muestra de docentes. (432) de una UPM. Nota. $n=$ muestra, $F=$ Estadístico de contraste.

\section{Fuentes de financiación.}

Instituto Tecnológico de Sonora (México).

\section{Referencias bibliográficas.}

Argudín, Y. (2005). Educación basada en competencias: nociones y antecedentes. México: Trillas.

Bosco, A. (2008). Las tecnologías de la información y la comunicación en la formación del profesorado: lineamientos, actualidad y prospectiva. Razón y Palabra, 63. Recuperado de http:// www.razonypalabra.org.mx/n63/abosco.html

Cabero, J. (2011). La competencia digital del profesorado: Un estudio en la Pontificia Universidad Católica Madre y Maestra. Sevilla: Fortic DC.

Consejo Federal de Educación (2010). Las políticas de inclusión educativa el programa conectar igualdad. Recuperado de http://www.me.gov.ar/consejo/ resoluciones/res10/123-10_01.pdf

Escudero, J. (1997). Tecnología Educativa: algunas reflexiones desde la perspectiva de la innovación y mejora de la educación. En C. Alonso (Coord.). La tecnología educativa la final del siglo XX: concepciones, conexiones $y$ límites con otras disciplinas. (pp. 33-47). Barcelona: Centre Telematic Editorial.

Fernández, C. \& Cebreiro, B. (2002). La preparación de los profesores para el dominio técnico el uso didáctico y el diseño/ producción de medios y nuevas tecnologías en Galicia. Revista de Innovación Educativa, 12, 109-122.

Lasnier, F. (2000). Réussir la formation par compétences. Montreal: Guerin.

López, A. (2003). Introducción de las nuevas tecnologías de la información (TICs) en la educación. Revista Boletín INFOLAC, 16(2). Recuperado de http://www.efdeportes.com/ efd156/sistema-de-informacion-web.htm

Marín, V., Vázquez, A.I., Llorente, M.C. \& Cabero, J. (2012). La alfabetización digital del docente universitario en el Espacio Europeo de Educación Superior. Edutec: Revista Electrónica de Tecnología Educativa, 39, 1-10. Recuperado de http://edutec.rediris.es/ $\mathrm{R}$ e v e 1 e c 2 / R e v e 1 e c 39 / p d f / Edutece_39_Marin_Vazquez_Llorente_Cabero.pdf

Píxel-Bit. Revista de Medios y Educación. No 44. Enero 2014. ISSN: 1133-8482. E-ISSN: 2171-7966. doi: http://dx.doi.org/10.12795/pixelbit.2014.i44.010 
Marquès, P. (2000). Nueva cultura, nuevas competencias para los ciudadanos: alfabetización digital. Recuperado de http:// www.peremarques.pangea.org/competen.htm

Ortega, J. (2008). Formación docente y uso escolar de las TIC. Boletín de Educación, 1(39), 28-36.

Pérez, R., Álvarez, C., del Moral, E. \& Pascual, A. (1998). Actitudes del profesorado hacia la incorporación de las nuevas tecnologías de la información en educación. En M. Cebrián de la Serna (Coord.). Recursos tecnológicos para los procesos de enseñanza yaprendizaje. (pp. 147-167). Málaga: Pirámide

Rangel, A. \& Peñalosa, E.A. (2013). Alfabetización digital en docentes de Educación Superior: construcción y prueba empírica de un instrumento de evaluación. Píxel-Bit. Revista de Medios y Educación, 43,9-23. doi: 10.12795/pixelbit.2013.i43.01

Rodés, V. (2011). Análisis de procesos de cambio tecnológico y organizacional para la integración de TIC en la universidad de la república. XIII Congreso Internacional de Educación a Distancia CREAD/ MERCOSUR/SUL 2009-UDEC. Santiago de Chile.

Rosario, H.J. \& Vásquez, L.F. (2012). Formación del docente universitario en el uso de TIC. Caso Universidades públicas y privadas (U. de Carabobo y U. Metropolitana). Píxel-Bit. Revista de Medios y Educación, 41, 163-171. Recuperado de http://acdc.sav.us.es/pixelbit/images/stories/ p41/12.pdf

Salinas, J. (2004). Innovación docente y uso de las TIC en la enseñanza universitaria. Revista universidad y sociedad del conocimiento, (RUSC), 1(1), 1-16.

Sanabria, A. (2006). Las TIC en el sistema escolar de Canarias: los programas institucionales de innovación educativa para la integración curricular de las Tecnologías de la Información y la Comunicación. RELATEC. Revista Latinoamericana de Tecnología Educativa, 5(2), 191-202.

Tejada, J. (2009). Competencias docentes: Profesorado. Revista de Currículum y Formación de Profesorado, 13(2), 1-15.

UNESCO (2008). Estándares de competencia en TIC para docentes. París. UNESCO.

Sierra, B. (1998). Técnicas de investigación social: Teoría y ejercicios. Madrid: Paraninfo

Vera, A. \& Torres, L. (2012). Características psicométricas de una medida de competencias básicas en TIC's para profesores de educación superior. Investigación Educativa Duranguense, 6(12), 40-45.

Fecha de recepción: 01-07-2013

Fecha de evaluación: 18-07-2013

Fecha de aceptación: 09-09-2013 